\title{
Estudo histológico da ossificação da cartilagem tireóidea
}

\section{Histologic study of ossification of the thyroid cartilage}

\author{
Walter P. Cruz¹, Rogério A. Dedivitis², \\ Ângelo Sementilli ${ }^{3}$, Abrão Rapoport
}

Palavras-chave: laringe, cartilagem tireóide, ossificação. Key words: larynx, thyroid cartilage, ossification.

\section{Resumo / Summary}

\section{C}

om o passar da idade, ocorrem progressivamente ossificação e formação de medula óssea nas cartilagens laríngeas. O conhecimento dessas modificações é importante para a diferenciação com invasão por câncer. Objetivo: Avaliar comparativamente as porções cranial e caudal da cartilagem tireóidea quanto a alterações histológicas em indivíduos adultos do sexo masculino, observando a ossificação e a formação de medula óssea. Forma de estudo: Experimental. Material e Método: Foram estudadas 7 laringes obtidas de cadáveres do sexo masculino submetidos a exame necrológico no Posto Médico-Legal de Santos, da Polícia Civil do Estado de São Paulo. A idade variou de 39 a 60 anos, com mediana de 53. Não se teve acesso à informação de hábito de tabagismo e etilismo. Os espécimes foram descalcificados e corados pela hematoxilina-eosina. A ossificação foi estudada pelo método histométrico baseado na estereologia e a presença de medula óssea foi realizada de forma qualitativa (presença ou ausência). Resultados: Nos segmentos craniais, notamos uma média de $42,8 \%$ de ossificação e a presença de medula óssea em $57,1 \%$. Nos segmentos caudais, a média de ossificação foi de $72,8 \%$, com medula óssea em $87,5 \%$. Conclusão: A ossificação da cartilagem tireóidea e a formação de medula óssea são mais intensas e precoces na sua topografia cranial.

\begin{abstract}
T he process of ossification and formation of bone marrow occurs in the larynx cartilages with age. The knowledge of this change is important for differentiation with cartilage invasion by cancer. Aim: Cranial and caudal segments of the thyroid cartilage were obtained from adult male larynges in order to evaluate comparatively the histological changes regarding to the ossification and the bone marrow formation. Study design: Experimental. Material and Method: Seven male adult larynges were obtained from cadavers underwent necrological examination in Posto Médico-Legal de Santos, da Polícia Civil do Estado de São Paulo. The ages ranged from 39 to 60 , with median of 53. There were no available data about to bacco and alcohol consumption. The specimens underwent decalcification and stained with $\mathrm{H}-\mathrm{E}$. Histometry was performed for quantitative analysis of the ossification while the bone marrow was analyzed according to qualitative criteria (presence or absence). Results: The average ossification was $42.8 \%$ and the bone marrow presence was observed in $57.1 \%$ in the cranial segments. In the caudal segments, the average ossification was $72.8 \%$, and the bone marrow was detected in $87.5 \%$ of the specimens. Conclusion: The ossification in the thyroid cartilage and the bone marrow formation increase and occur earlier moving downward from the cranial to the caudal aspects.
\end{abstract}

Mestre pelo Curso de Pós-Graduação em Ciências da Saúde do Hospital Heliópolis HOSPHEL, São Paulo

2 Doutor em Medicina pelo Curso de Pós-Graduação em Otorrinolaringologia e Cirurgia de Cabeça e Pescoço da Universidade Federal de São Paulo - Escola Paulista de Medicina, São Paulo. Professor do Curso de Pós-Graduação em Ciências da Saúde do Hospital Heliópolis HOSPHEL, São Paulo.

${ }^{3}$ Mestre em Anatomia Patológica pela Universidade Federal de São Paulo - Escola Paulista de Medicina, São Paulo; Chefe do Serviço de Anatomia Patológica do Hospital Ana Costa, Santos.

${ }^{4}$ Professor Livre Docente em Cirurgia de Cabeça e Pescoço pela Universidade de São Paulo. Coordenador do Curso de Pós-Graduação em Ciências da Saúde do Hospital Heliópolis HOSPHEL, São Paulo.

Curso de Pós-Graduação em Ciências da Saúde do Hospital Heliópolis HOSPHEL, São Paulo.

Endereço para Correspondência: Walter Paiva Cruz - Rua Dr. Olinto Rodrigues Dantas 343 cj. 62 Santos SP 11050-220 Tel (0xx13)3232-9292 - Fax (0xx13)3237-6375 - E-mail: wpcruz@uol.com.br

Artigo recebido em 28 de março de 2003. Artigo aceito em 11 de setembro de 2003. 


\section{INTRODUÇÃO}

A avaliação pré-operatória de pacientes com carcinoma de laringe é fundamental para a indicação da terapêutica mais adequada. A combinação do estudo endoscópico e radiológico permite realizar o estadiamento do tumor e detectar a invasão das cartilagens da laringe. Muitas informações importantes sobre o carcinoma da laringe podem ser obtidas pela endoscopia, entretanto a invasão profunda do tumor só pode ser estimada. A tomografia computadorizada pode detectar se há ou não invasão das cartilagens, porém existe certa dificuldade por parte dos radiologistas em determinar a diferença radiológica entre invasão tumoral e ossificação endocondral.

A invasão da cartilagem por tumor pode contra-indicar uma ressecção parcial, no entanto, o processo de ossificação e de formação de medula óssea pode mimetizar tal infiltração. Assim, o conhecimento preciso da localização dos pontos de ossificação da cartilagem tireóidea pode ajudar no diagnóstico e, portanto, na conduta terapêutica ${ }^{1}$.

O objetivo deste trabalho consiste em avaliar comparativamente as alterações histológicas que ocorrem na cartilagem tireóidea em indivíduos adultos do sexo masculino no que diz respeito à ossificação e à formação de medula óssea nas suas porções cranial e caudal.

\section{MATERIAL E MÉTODO}

Estudamos, após prévia autorização do Núcleo de Perícias Médico-Legais de Santos/SP, laringes obtidas de sete cadáveres submetidos a exame necrológico no Posto MédicoLegal de Santos, da Polícia Civil do Estado de São Paulo. A idade variou de 25 a 63 anos, com mediana de 53 e média de 50 anos. Os espécimes estudados provinham de pessoas indigentes, adultas, do sexo masculino, entre 8 e 24 horas após o óbito. Foram excluídos espécimes de cadáveres cuja causa do óbito tivesse relação direta com a laringe ou que tenham sido manipulados por intubação laringo-traqueal ou quando se apresentassem em início de putrefação. Não se teve acesso à informação de hábito de tabagismo e etilismo dos indivíduos.

Para cada cadáver, respeitamos as incisões habitualmente realizadas para o exame necrológico. Dissecada e exposta a laringe, fizemos uma inspeção inicial, permitindo verificar ausências de lesões macroscópicas da mucosa e cartilagens. Retirada a musculatura extrínseca e o pericôndrio externo da cartilagem tireóidea, com o uso de uma serra elétrica, removemos um pequeno fragmento de cartilagem tireóidea de espessura total de $5 \times 5$ milímetros da porção cranial e outro fragmento de mesmo tamanho da porção caudal da lâmina (Figura 1).

Os fragmentos foram colocados separadamente em um frasco com formol e enviados para o laboratório de anatomia patológica para exame microscópico. A descalcificação foi obtida colocando-se o espécime em solução de ácido fórmico a $5 \%$, por um perío do que variou de três a cinco dias. 0 patologista que realizou a avaliação não conhecia de qual topografia da lâmina cada fragmento foi retirado e foi realizada a coloração por hematoxilina-eosina.

Para o estudo da ossificação foi utilizado o método histométrico baseado nos princípios de estereologia que, pela análise quantitativa, fornece valores percentuais dos componentes analisados no plano bidimensional, proporcionando, assim, uma noção do que ocorre no plano tridimensional ${ }^{2,3}$. Para atingir tais valores, foi utilizada uma ocular integradora I Kpl-W10X da Zeiss, com retículo contendo 25 pontos distribuídos geometricamente. Os valores obtidos expressaram uma proporção e não uma medida absoluta. 0 retículo projetado sobre os cortes permitiu a contagem de "hits", ou seja, dos pontos que incidiram nos tecidos ósseo e cartilaginoso. Este procedimento foi realizado com objetiva de 40X pelo médico patologista e, para cada espécime, foram contados dez campos.

A análise da presença de medula óssea foi realizada de forma qualitativa, ou seja, foi considerada a presença ou ausência em cada espécime estudado.

\section{RESULTADOS}

Nas regiões com cartilagem hialina típica, notaram-se condrócitos típicos, mais achatados próximo à superfície articular, mais arredondados na profundidade (centro da cartilagem) e alguns agregados, formando grupos isógenos. A ossificação, quando observada, ocupava apenas uma área bem localizada das cartilagens ou então a substituição era total, com presença apenas de tecido ósseo lamelar típico, com esboços de sistemas de Havers. Na primeira situação, o tecido ósseo presente era do tipo lamelar, sendo as lamelas paralelas à superfície das cartilagens. No centro do tecido ósseo, observamos, em alguns casos, a presença de uma cavidade óssea preenchida por tecido adiposo ou medula óssea com tecido hematopoiético.

A Tabela 1 representa idade, porcentagem de tecido ósseo encontrado e presença ou não de medula óssea nos aspectos cranial e caudal de cada cartilagem estudada. Nos segmentos craniais, notamos que três casos não apresentaram ossificação nem medula óssea. Os outros quatro casos apresentaram ossificação, cuja presença variou de 30 a $100 \%$ (Figura 2). Em todos estes quatro casos, observou-se a presença de medula óssea. A média de ossificação nos sete casos foi de $42,8 \%$. Já a presença de medula óssea foi observada em quatro dos sete casos $(57,1 \%)$. Nos segmentos caudais, apenas um caso não apresentou ossificação nem medula óssea. A ossificação esteve presente em todos os outros, variando de 30 a $100 \%$ (Figura 3), sendo que quatro apresentaram $100 \%$ de ossificação. A média dos sete espécimes foi de $72,8 \%$ de ossificação. A presença de medula óssea foi observada em seis dos sete casos $(87,5 \%)$. 
Q uanto à análise estatística, para a caracterização da amostra, utilizaram-se estatísticas descritivas. As variáveis categóricas foram representadas por freqüências absolutas e relativas e foram utilizadas medidas de tendência central e de variabilidade para as variáveis contínuas (Tabela 2).

\section{DISCUSSÃO}

As cartilagens tireóidea, cricóidea e aritenóidea são do tipo hialina e a epiglote, corniculada, cuneiforme e 0 ápice da aritenóide são do tipo elástica. Com a idade, as cartilagens elásticas têm pequena tendência para calcificação, mas não ossificação, no entanto, as cartilagens hialinas tornamse mais ou menos ossificadas ${ }^{4}$. A cartilagem tireóidea sofre

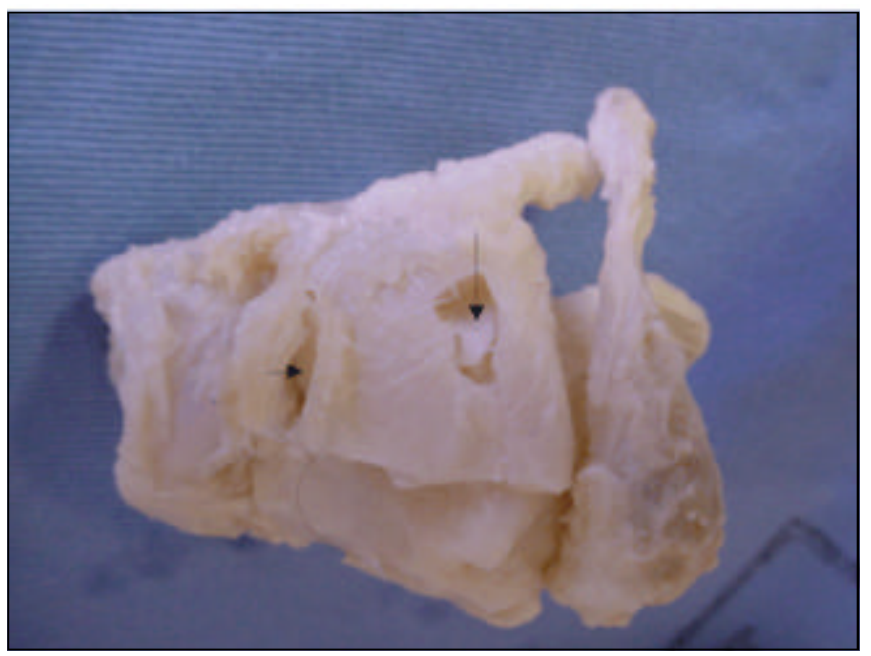

Figura 1. Espécime anatômico estudado com as janelas cranial (seta maior) e caudal (seta menor) na asa da cartilagem tireóidea de onde foram obtidos fragmentos para estudo histológico.

Tabela 1. Porcentagem de tecido ósseo encontrado e presença de medula óssea nos espécimes

\begin{tabular}{|c|c|c|c|c|c|}
\hline \multirow[t]{2}{*}{ Caso } & \multirow[t]{2}{*}{ Idade } & \multicolumn{2}{|c|}{ Porção cranial } & \multicolumn{2}{|c|}{ Porção caudal } \\
\hline & & $\begin{array}{c}\text { Ossificação } \\
(\%)\end{array}$ & $\begin{array}{l}\text { Presença } \\
\text { de medula }\end{array}$ & $\begin{array}{c}\text { Ossificação } \\
(\%)\end{array}$ & $\begin{array}{l}\text { Presença } \\
\text { de medula }\end{array}$ \\
\hline 1 & 57 & 100 & + & 100 & + \\
\hline 2 & 39 & 30 & + & 80 & + \\
\hline 3 & 53 & 0 & 0 & 0 & 0 \\
\hline 4 & 63 & 80 & + & 100 & + \\
\hline 5 & 52 & 0 & 0 & 100 & + \\
\hline 6 & 60 & 90 & + & 100 & + \\
\hline 7 & 25 & 0 & 0 & 30 & + \\
\hline
\end{tabular}

Legenda: + - presença de medula óssea; 0 - ausência de medula óssea ossificação endocondral com o passar do tempo. 0 grau de ossificação é menor no sexo feminino e ocorre uma simetria de ossificação na cartilagem tireóidea em ambos os sexos ${ }^{5}$.

A cartilagem tireóidea hialina apresenta progressivo aumento de fibras colágenas e mineralização. 0 processo de mineralização leva à calcificação ou à ossificação ${ }^{6}$. 0 processo de disseminação da ossificação ocorre diferentemente entre os sexos e até entre pessoas do mesmo sexo e este processo está relacionado com a idade de cada indivíduo ${ }^{7}$.

Os fenômenos de calcificação e ossificação devem ser diferenciados. Independentemente dos raros casos de verdadeira infiltração calcária, o cálcio deposita-se geralmente em zonas previamente alteradas por outras causas. Esse fato é apoiado pelas várias descrições patológicas de calcificação

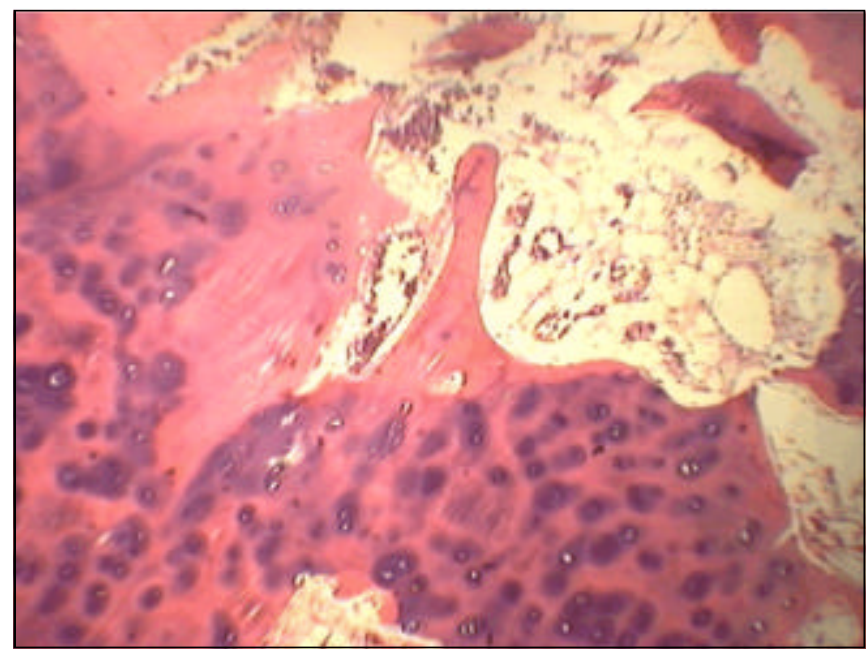

Figura 2. Corte de fragmento cranial de cartilagem tireóidea que revela cartilagem nas margens e início de formação de lamelas ósseas no centro (HE 40x).

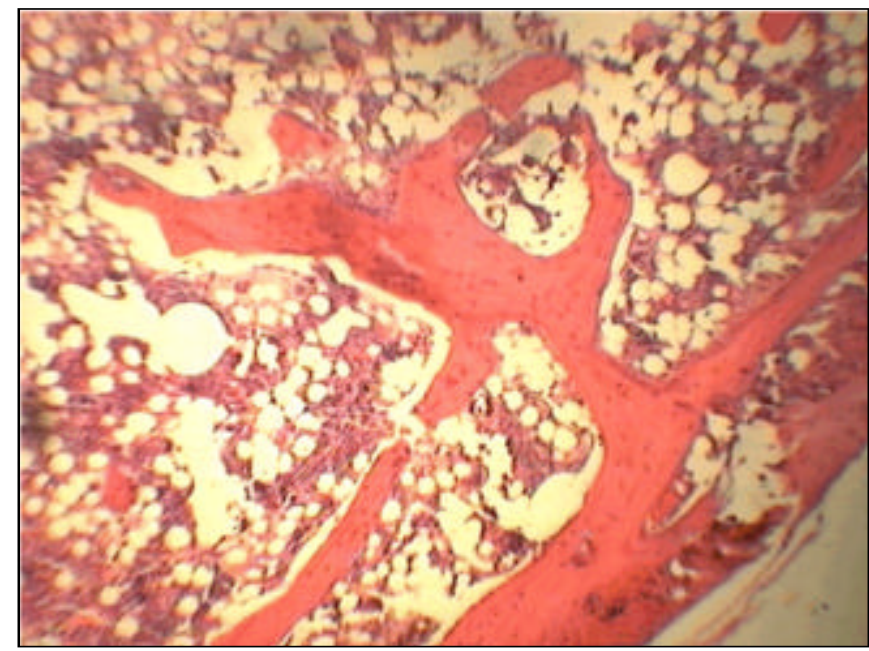

Figura 3. Corte de fragmento caudal de cartilagem tireóidea mostrando tecido ósseo formado por lamelas uniformes e região medular com células hematopoiéticas (HE 40x). 
Tabela 2. Distribuição dos sete casos de acordo com as porções cranial e caudal

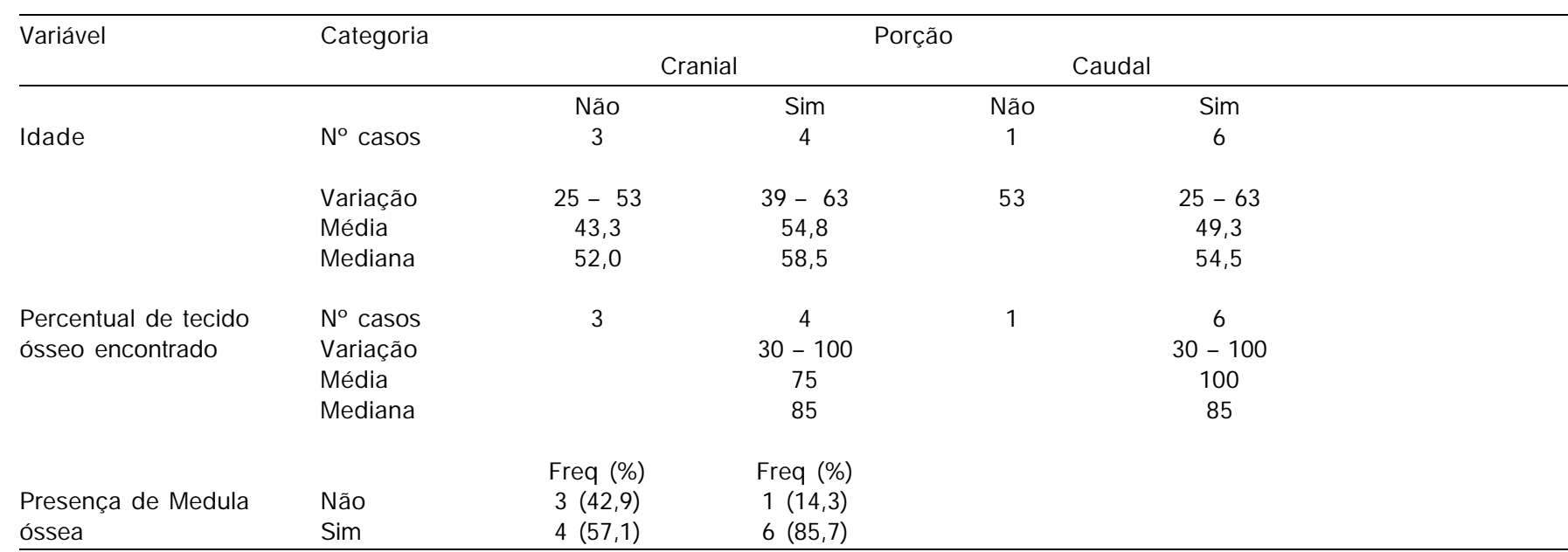

Legenda: Não - ausência de ossificação e de medula óssea: sim - presença de ambos.

de partes necróticas de vários órgãos. 0 fenômeno é mais observado após os sessenta anos de idade ${ }^{8}$. Na maior parte dos casos, a deposição ocorre na substância fundamental onde é mais intensa a morte de muitas células. Já a ossificação é mais freqüente no sexo masculino e após os quarenta anos de idade. A zona ossificada apresenta a estrutura de típico osso lamelar. 0 osso é sempre delimitado por um halo de cartilagem, nunca haven do uma zona de transição, o que confirma que o processo é de substituição ${ }^{9}$.

O cálcio deposita-se em ilhas, havendo regiões densamente calcificadas pela matriz. 0 grau de diferenciação dos condrócitos do pericôndrio diminui eas células localizadas mais profundamente mostram sinais de perda de viabilidade, surgem focos de erosão e o ritmo de surgimento dessas alterações acelera-se com 0 envelhecimento ${ }^{10-12}$, não se observando nos neonatos e na infância ${ }^{13}$. A osteogênese parece surgir em função da força de tensão a que se submete a articulação, como em estímulos causados pelas contrações musculares ${ }^{14}$. Além disso, distúrbios do metabolismo mineral e outros processos patológicos degenerativos, como a artrite reumatóide, têm impacto na osteogênese.

A ossificação tem início na região subcortical, havendo envolvimento na porção central somente nos espécimes dos mais idoso ${ }^{15}$. A ossificação das cartilagens inicia-se apenas quando está completo 0 crescimento do esqueleto ${ }^{16}$. Há possibilidade também de que estímulos das contrações dos músculos extrínsecos e intrínsecos da laringe tenham um efeito mecânico de tensão e pressão, contribuindo para ossificação ${ }^{17}$. Os casos de achado mais precoce de calcificação são no final de segunda década ${ }^{18}$. 0 padrão de calcificação é similar entre gêmeos idênticos, o que indica uma determinação hereditária ${ }^{19}$.

0 estudo por ressonância nuclear magnética de laringes removidas de cadáveres mostrou a presença de tecido hematopoiético nas cartilagens hialinas ossificadas. Ossificação e sinais de tecido medular são observados a partir dos trinta anos de idade ${ }^{20}$. É por esta razão que optamos por realizar nossa investigação em grupo etário constituído por adultos, ou seja, em indivíduos já passíveis de sofrer as referidas alterações histológicas, mas não tão idosos a ponto de ter uma substituição quase total por tecido ósseo em ambas as topografias estudadas.

A importância da ossificação revela-se tanto na avaliação radiológica de tumores como na realização das laringectomias parciais, já que a infiltração da cartilagem tireóidea torna o estadiamento do tumor primário como $\mathrm{T}_{4}$. Os tumores apresentam freqüentemente densidade similar às porções de cartilagens que não sofreram ossificação. No entanto, a ausência de ossificação próxima ao tumor poderia sugerir que a cartilagem foi invadida. Invasão por tumores pode ainda ser confundida com a presença de medula óssea, que pode mimetizar área de infiltração ${ }^{21}$. Tumores não invadem a cartilagem tireóidea simetricamente como ocorrem na ossificação, portanto, os pontos de ossificação devem ser conhecidos para não se interpretar erradamente a tomografia computadorizada.

Estudo tomográfico de laringes obtidas de cadáver revelou uma tendência a maior ossificação a medida que se caminha no sentido crânio-caudal da cartilagem tireóidea ${ }^{1}$. Estudo por radiografia simples revelou que a ossificação desta cartilagem ocorre nos sentidos dorso-ventral e caudo-cranial ${ }^{5}$. Nossos achados coincidem com estes relatos.

\section{CONCLUSÃO}

A análise do material estudado permite concluir que ocorre, em adultos do sexo masculino, um processo de ossificação e de formação de medula óssea com tecido 
hematopoiético típico dentro da cartilagem tireóidea, sendo mais intenso na topografia caudal. Tal conhecimento tem impacto na avaliação radiológica de pacientes com câncer de laringe e na programação terapêutica, inclusive quanto à realização de laringectomia parcial.

\section{REFERÊNCIASBIBUOGRÁFICAS}

1. Yeager VL, Lawson C, Archer CR. Ossification of the laryngeal cartilages as it relates to computed tomography. Invest Radiol 1982;17:11-9.

2. Underwood EE. Stereology or the quantitative evaluation of microstructure. J Microsc (Oxf.) 1968; 89:161-90.

3. Weibel ER, Kistler GS, Scherle WF. Practical stereological methods for morphometric cytology. J Cell Biol 1970; 30:23-38.

4. Milroy CM. Ossification of the epiglottis. J Laryngol Otol 1992; 106:180-2.

5. Jurik AG. Ossification and calcification of the laryngeal skeleton. Acta Radiol Diagn (Stockh.) 1984; 25:17-22.

6. Strek P, Nowogrodzka-Zagorska M, Skawina A, Olszewski E. Ossification of human thyroid cartilage. Scanning electron microscopic study. Folia Morphol (Warsz.) 1993; 52:47-56.

7. Zeligman SB. Calcification and ossification of thyroid cartilage in men. Arch Anat Histol Embriol 1960;9:97-102.

8. Dedivitis RA, Abrahão $M$, Simões MJ, Mora AO, Cervantes 0 . Cricoarytenoid joint: histologic changes during aging. São Paulo Med J 2001; 119:89-90.

9. Amprino $R$, Bairati A. Studi sulle trasformazione delle cartilagini Del I'uomo nell'accrescimento e nella senescenza. Z Zellforsch Mikrosk Anat 1933;20:143-205.

10. Hass GM. Studies of cartilage IV. A morphologic and chemical analysis of aging human costal cartilage. Arch Pathol 1943; 35:275-84.
11. Kahane JC, Hammons J. Developmental changes in the articular cartilage of the human cricoarytenoid joint. In: Baer T, Harris K, Sasaki C. Vocal Physiology. San Diego: College Hill Press; 1987. p. 14-28.

12. Paulsen FP, Tillmann B. Composition of the extracellular matrix in human cricoarytenoid joint articular cartilage. Arch Histol Cytol 1999; 2:149-63.

13. Hirano M, Sato K. Histological Color Atlas of the Human Larynx, $1^{a}$ Edição. San Diego: Singular Publishing Group, Inc.; 1993.

14. Thawley SE. Surgical therapy of the larynx: surgical anatomy in Thawley SE, Panje WR, Batsakis JG, Lindberg RD. Comprehensive

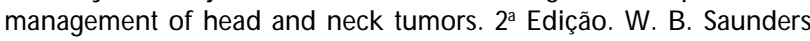
Company; 1999.

15. Casiano RR, Ruiz PJ, Goldstein W. Histopathologic changes in the aging human cricoarytenoid joint. Laryngoscope 1994; 104: 5338.

16. Chiewitz S. Untersuchungen über die veränderungen der menschlichen kehlkopfknorpel. Arch $f$ Anat u Entweklngsgesch 1882; 303-49.

17. Strek P, Skawina A, Petri M, Olszewski E, Nowogrodzka-Zagorska $\mathrm{M}$, Waloch J. The investigations of mechanical properties of ossificationg thyroid cartilages of the human Iarynx. Folia Morphol (Warsz.) 1993; 52:39-46.

18. Hately W, Evison G, Samuel E. The pattern of ossification in the laryngeal cartilages: a radiological study. Brit J Radiol 1965; 38: 585-91.

19. Vastine II JH, Vastine MF. Calcification in the laryngeal cartilages. Arch Otolaryngol 1952; 55:1-7.

20.Sakai F, Gamsu G, Dillon WP, Lynch DA, Gilbert TJ. MR Imaging of the larynx at $1.6 \mathrm{~T}$ J Comp Assist Tomogr 1990; 14:60-71.

21. Kavanagh KT, Salazar JE, Babin RW. Bone marrow expansion of the thyroid cartilage: A source of confusion with malignant invasion in CT studies. J Comp Assist Tomogr 1985; 9:177-9. 